\title{
The effect of task-specific balance training program in dual-task and single- task conditions on balance performance in children with developmental coordination disorder
}

\author{
Hamideh Jahanbakhsh' ${ }^{1}$, Mehdi Sohrabi ${ }^{1, *}$, Alireza Saberi Kakhki ${ }^{1}$, and Ezzat Khodashenas ${ }^{2}$ \\ ${ }^{\text {I}}$ Faculty of Sport Sciences, Ferdowsi University of Mashhad, Mashhad, Iran; and ${ }^{2}$ Faculty of Medicine, Mashhad University \\ of Medical Sciences, Mashhad, Iran
}

Copyright: (C) $2020 \mathrm{H}$. Jahanbakhsh et al. This is an open access article licensed under the Creative Commons Attribution License (https://creativecommons.org/licenses/by/4.0/).

\begin{abstract}
Background: Among the main deficits in children with developmental coordination disorder (DCD), the underdeveloped balance is important because of the impact on motor skills development, the falling risks and participation in various physical activities. Objective: The purpose of this study was to examine the effect of task-specific balance training in dual-task and single-task conditions on balance performance in children with DCD. Methods: Thirty-nine boys aged from 7 to 9 years with DCD were randomly assigned to 3 groups: dual-task training group $(n=13$, mean age $8.59 \pm 0.82$ years $)$, single-task training group $(n=13$, mean age $8.61 \pm 0.83$ years $)$ and control group without any intervention ( $n=13$, mean age $8.41 \pm 0.86$ years). Each session in the single-task or dual-task group continued for 45 minutes, three times per week for a duration of 8 weeks. The balance of all three groups was measured using the Stork Balance Stand Test and dynamic Y Balance Test in the pre-test, post-test (after 24 sessions), and follow-up phases ( 2 months after the intervention). Results: There was a significant difference between the dual-task and singletask training program in static and dynamic balance tests score during post-test. The dual-task program significantly improved the balance performance of children with DCD $(p<.001)$. Conclusions: Based on the findings of the present research, the dual-task training condition focusing on balance and cognitive tasks may improve the skills of children on performing static and dynamic balance significantly more than the single-task training.
\end{abstract}

Keywords: static balance, dynamic balance, developmental coordination disorder, dual-task

\section{Introduction}

Developmental coordination disorder (DCD) is a common movement disorder of childhood, with a prevalence rate of around 5-6\% in school-aged children (Smits-Engelsman et al., 2018). DCD occurs when a delay in the development of motor skills, or problem with coordinating movements, causes the child to be unable to perform normal or daily tasks. By definition, coordination problems of children with DCD are not associated with an identifiable medical or neurological condition. These motor difficulties are apparent from an early age (Caçola \& Lage, 2019).

These children are determined by poor gross motor control and impaired postural control that may

\footnotetext{
* Address for correspondence: Mehdi Sohrabi, Department of Motor Behavior, Faculty of Sport Sciences, Ferdowsi University of Mashhad, Azadi Square, Mashhad, 9177948974, Iran. E-mail: sohrabi@um.ac.ir
}

interfere with daily activities of life and participation in sport (Speedtsberg et al., 2017).

The results of studies show that disturbances in balance are one of the most common sensorimotor deficits that $73-87 \%$ of children with DCD face it (Fong, Guo, Liu, et al., 2016). Poor balance has an important role in the DCD-related motor impairments (Speedtsberg et al., 2017), so without appropriate interventions, problems of this group of children may transfer to adolescence and adult stages (Giagazoglou, Sidiropoulou, Mitsiou, Arabatzi, \& Kellis, 2015). Therefore, evidence-based treatment strategies to improve balance control in this group of children need to be advanced (Fong, Guo, Cheng, et al., 2016).

New approaches adopted to improve balance in DCD children primarily focus on triggering plasticity in the central nervous system by applying the taskoriented treatment (Fong, Guo, Cheng, et al., 2016). At the present time, "task-oriented" approaches are the most common treatment methods for improving 
motor skills and consequently, balance ability of the DCD children. This treatment approach is based on principles of motor learning and neural formation (plasticity; Zwicker, Missiuna, Harris, \& Boyd, 2012).

Researchers have recently developed a promising treatment strategy by adopting the task-oriented approach titled "task-specific intervention". This approach is based on dynamical systems theory and principles of coordination dynamics (P. H. Wilson, 2005).The taskspecific intervention focuses on the direct teaching of the task to be learned. It is based on the premise that performance is the result of learning and that learning is optimal when teaching is focused directly at the target task (Mandich, Polatajko, Macnab, \& Miller, 2001). The key element of this treatment approach lies in the repeated exposure of the child to a particular task (e.g., a balance task) with the right constraints (e.g., the child's natural environment; P. H. Wilson, 2005).

A number of research findings have indicated that the task-specific intervention has the potential to improve motor and different balancing skills of DCD children (Fong, Guo, Cheng, et al., 2016; Fong, Guo, Liu, et al., 2016; Revie \& Larkin, 1993).

Considering the fact that ecological or motor-cognitive views focus on the use of cognitive skills in their interventional programs (Sugden, 2007), the majority of the researches adopt one-dimensional and a singletask program for DCD children. contrary to the fact that individuals perform dual-tasks in their ordinary life activities intentionally or unconsciously (Elhinidi, Ismaeel, \& El-Saeed, 2016). This overlapping type of activities is called a "dual-task" (Andersson, Hagman, Talianzadeh, Svedberg, \& Larsen, 2002). As an experimental design, two tasks are performed simultaneously; one named the primary and the second one called the secondary task. During the execution of the secondary task, the performance level of the first task remains at the baseline level. In this case, if the performance level of the secondary task deteriorates, it indicates that the primary task requires more attention (Choi, Kim, Han, $\&$ Kim, 2015). Despite the fact that normal individuals show no difficulties in performing these kinds of tasks (Choi et al., 2015), the DCD children demonstrate difficulties when performing dual-tasks (Cherng, Liang, Chen, \& Chen, 2009). In fact, these children show considerable variability in their motor performances (e.g., walking, foot-hand coordination). This type of variability may result in less efficient automaticity of performing motor tasks or delayed automaticity in the development of these motor skills in DCD children. Reduced automaticity in motor tasks leads to an increased demand for attention which in turn results in poor performance of DCD children during the performance of a dual-task (Cherng et al., 2009).
The results of studies on adult and elderly patients suffering from balance disorders indicate that performing simultaneous stability and cognitive tasks has a significant effect on balance and muscle coordination involved in balance control (Makizako et al., 2012). Furthermore, Cherng et al. (2009) demonstrated that performing dual-tasks requiring balance compared to single-tasks requiring balance resulted in more improvement of dynamic balance in ADHD children (Chen et al., 2012). The implications of these findings are that single-tasks require less processing demands compared to dual-tasks. In other words, during the dual-task, cognitive and motor demands result in improvement of neuroplasticity and establishment of new neural associations (Wild et al., 2013) which significantly improve balancing functions and motor coordination (Hamacher, Hamacher, \& Schega, 2014).

Considering the significance and benefits of this training method, the majorities of the existing research findings on DCD children focus on single-task applications. While the benefits of employing dual-task training for adult patients with balance disorder is partly confirmed, it is still unclear whether these findings can be generalized to other age groups including children with DCD disorder. Thus, assess whether these children may also benefit from a specific dual-task training program to improve their balancing skill requires a carefully designed research protocol. In our study, we focused on boys due to their availability. So, the aim of this study was to examine the effect of task-specific balance training in dual-task and single-task conditions on balance performance in boys with DCD.

\section{Methods}

\section{Participants}

The sample size for the research design was determined by applying PASS software (Version 2008; NCSS, Kaysville, UT, USA). Considering the statistical power of .80 with an alpha level set to .05 for a two-tailed test, a sample size of 39 participants was calculated. These children were selected from the 7-9-year-old diagnosed DCD children of elementary schools of the city of Ahwaz. The inclusion criterion included: (1) a formal diagnosis of DCD, (2) age range of 7-9 years, and (3) normal IQ (above 70). The exclusion criterion was the diagnosis of the autism spectrum, attention deficit hyperactivity disorder, neurological or physical disorders and prescription of any medication, occupational therapy treatment and lack of regular participation in physical activity.

The DCD children were selected based on four criteria set by the DCD criteria of the Diagnostic and 
Statistical Manual of Mental Disorders; including a score $\leq 16$ th percentile on the Bruininks-Oseretsky Test of Motor Proficiency (criterion A), clear interference of motor difficulties with ordinary life activities (criterion $\mathrm{B}$, scoring $\leq 15$ th percentile on the Developmental Coordination Disorder Questionnaire Version 7 [DCDQ'07] completed by parents), the onset of movement difficulties from the primary stage of development (criterion $\mathrm{C}$ ), and if other disorders such as neurological, mental disabilities, visual defects, or muscular dystrophy were detected (criterion D; SmitsEngelsman, Schoemaker, Delabastita, Hoskens, \& Geuze, 2015). For criterion C and D, children were referred to a psychiatrist.

After the completion of the selection stage, the participants were randomly assigned into three groups; single-task $(n=13)$, dual-task $(n=13)$ and control ( $n=13$, without participation in any intervention) groups. Written informed consent was obtained from the parents and ethical committee of Ferdowsi University of Mashhad for biological researches approved the protocol (IR.MUM.FUM.REC.1397.12). All the procedures were fully explained to the participants and their parents.

\section{Interventions}

The training protocol used for this research included physical exercise to improve static and dynamic balance; the exercise activities were progressively repetitive dynamic and static balance movements in which the orderly stage level was applied. The training program was designed based on the task-oriented approach with an emphasis on improving balancing skills which were primarily performed in a closed environment. Then as the training programs advanced, the movements became more complicated in an open environment. Gentile's two-dimensional classification system was adopted in designing the tasks (Magill, 2007). The training programs were performed for 45 minutes, three times per week for a duration of 8 weeks. Overall, the training program consisted of 24 sessions, which were performed in a gymnasium under the supervision of one skilled trainer and two master degree physical education trainers. Challenging but exciting physical activities were selected to increase the motivation of children. The exercise programs were very repetitive in nature.

The training program included 10 minutes of warm-up followed by 30 minutes of main activities and 5 minutes of cooling down. In the single-task group, the child was instructed to keep balance while standing on one leg, maintain balance while walking. Indeed, children performed the designed balance program. The balance activities were selected which could be simply performed at home or school without any facilities or apparatus (Table 1). These selected tasks were used by some previous studies (Cheldavi, Shakerian, Boshehri, \& Zarghami, 2014; Fong, Guo, Cheng, et al., 2016;

Table 1

Descriptions of the exercises that comprised the balance training intervention (single-task group)

\begin{tabular}{|c|c|c|c|}
\hline Balance exercise & Description & Repetitions/sets & Progression level \\
\hline Double leg stance & $\begin{array}{l}\text { Maintain balance with bare feet in } \\
\text { different conditions }\end{array}$ & 3 repetitions of $10 \mathrm{~s}$ & $\begin{array}{l}\text { Level 1: stand on full feet, eyes closed } \\
\text { Level 2: stand on toes, eyes open } \\
\text { Level 3: stand on toes, eyes closed } \\
\text { Level 4: stand on toes, eyes closed, keep an } \\
\text { object in hands }\end{array}$ \\
\hline $\begin{array}{l}\text { Single leg stance } \\
\text { (alternate feet) }\end{array}$ & $\begin{array}{l}\text { Maintain balance in upright unilat- } \\
\text { eral stance }\end{array}$ & $\begin{array}{l}3 \text { repetitions of } 10 \mathrm{~s} \\
\text { on each leg }\end{array}$ & $\begin{array}{l}\text { Level 1: hard surface, eyes open } \\
\text { Level 2: hard surface, eyes closed } \\
\text { Level 3: foam, eyes open } \\
\text { Level 4: foam, eyes closed }\end{array}$ \\
\hline Balance path & $\begin{array}{l}\text { Walk in a straight line }(4.5 \mathrm{~m}) \text { with } \\
\text { heels raised. Progress to heel-to-toe } \\
\text { walk in a straight line }(4.5 \mathrm{~m})\end{array}$ & $\begin{array}{l}3 \text { trips (back and } \\
\text { forth) }\end{array}$ & $\begin{array}{l}\text { Level 1: linear path, gait } \\
\text { Level 2: curved path, gait } \\
\text { Level 3: linear path, heel-toe } \\
\text { Level 4: curved path, heel-toe }\end{array}$ \\
\hline $\begin{array}{l}\text { Forward hop on } \\
\text { marking sheets } \\
\text { (alternate feet) }\end{array}$ & $\begin{array}{l}\text { Double-leg hop or jump forward. } \\
\text { Progress to single-leg hop forward }\end{array}$ & $\begin{array}{l}3 \text { sets of } 10 \text { repeti- } \\
\text { tions on each leg }\end{array}$ & $\begin{array}{l}\text { Level 1: jump on marking sheets } \\
\text { Level 2: hop on marking sheets. } \\
\text { Level 3: jump on marking sheets, keep an } \\
\text { object in hand } \\
\text { Level 4: hop on marking sheets, keep an } \\
\text { object in hand }\end{array}$ \\
\hline
\end{tabular}

Note. Frequency was three times per week. Each exercise was advanced through progression levels according to each participant's skilled ability and willingness to be challenged by more difficult forms of an exercise. 
Fong, Guo, Liu, et al., 2016; Strang, Haworth, Hieronymus, Walsh, \& Smart, 2011).

The dual-task program was very similar to the singletask program with the exception that the participants in the dual-task group were required to perform cognitive tasks such as counting numbers, remembering a collection of letters, describing pictures or naming different objects, or making discriminatory judgments while executing the designed balance program (Tables 2 and 3 ). These tasks were modified versions of tasks used by some previous studies (Plummer-D'Amato et al., 2012; Silsupadol, Siu, Shumway-Cook, \& Woollacott, 2006). The control group performed their regular daily tasks.

\section{Anthropometric data}

Anthropometric characteristics of the children including standing height, weight, leg length (from the anterior superior iliac spine to the inferior distal surface of the medial malleolus) were measured.
Modified version of the parent's DCDQ'07 This test was employed as a tool to diagnose DCD for children aged 5-15 years old. Based on the guideline of scoring this instrument, if the children's total score was less than 46 (between the age 5 to 7 years and 11 months old), less than 55 (between the age 8 to 9 years and 11 months old), they are diagnosed as a potential suspect of facing identifiable of DCD. B. N. Wilson et al. (2009) reported the sensitivity and specificity of this instrument as .84 and .87 , respectively.

\section{Bruininks-Oseretsky Test of Motor Proficiency}

This test is composed of several subtests that measure reference norms for the motor performance of children 4.5 to 14.5 years old. The complete set of this test included 8 subscales that evaluate the fine and gross motor skills or motor disorders (Bruininks, 1978). The validity of this test is .84 reported by BruininksOseretsky (Bruininks \& Bruininks, 2005). The test was

Table 2

Secondary tasks in training programs for dual- task group

\begin{tabular}{|c|c|}
\hline Cognitive training & Progression level \\
\hline Name things/words & $\begin{array}{l}\text { Expression of simple words (animals, fruits, colors, clothes) } \\
\text { Expression of difficult words (cities, provinces, provincial capital, names of men and women, flowers) } \\
\text { Expression of words starting with a specific letter }\end{array}$ \\
\hline Remembering things & $\begin{array}{l}\text { Remembering the path from home to lab } \\
\text { Remembering of certain names/numbers from the list } \\
\text { Visual discrimination tasks: participants were shown the pictures before and after performing the balance } \\
\text { tasks. They were asked to memorize the pictures and to respond if the pictures were the same. They were } \\
\text { required to say "yes" if the pictures were the same, and "no" if they were different. }\end{array}$ \\
\hline Counting numbers & $\begin{array}{l}\text { Direct number counting } \\
\text { Backward number counting } \\
\text { Random number counting }\end{array}$ \\
\hline N-Back task & $\begin{array}{l}\text { Reverse 3-letter words or 3-digit numbers } \\
\text { Reverse 4-letter words or 3-digit numbers } \\
\text { Reverse the days of the week, months of the year and directions }\end{array}$ \\
\hline
\end{tabular}

Table 3

Descriptions of the exercises that comprised the balance-cognitive training intervention (dual-task group)

\begin{tabular}{lll}
\hline Balance task & Cognitive task (secondary tasks) & Focus (balance/cognitive task) \\
\hline Stand on full feet, eyes closed & Count backward by 3 & $80 / 20$ (emphasis on balance task) \\
Stand on full feet, eyes closed & Count backward by 3 & $20 / 80$ (emphasis on cognitive task) \\
Stand on toes in double leg stance, eyes open & Name any words starting with letter A-K & $80 / 20$ (emphasis on balance task) \\
Stand on toes in double leg stance, eyes open & Name any words starting with letter L-X & $20 / 80$ (emphasis on cognitive task) \\
Walking in linear path, heel-toe & Remember words & $80 / 20$ (emphasis on balance task) \\
Walking in linear path, heel-toe & Remember words & $20 / 80$ (emphasis on cognitive task) \\
Walking in curved path, heel-toe & Visual imaginary task (tell the road direc- & $80 / 20$ (emphasis on balance task) \\
& tion from home to the lab) & \\
Walking in curved path, heel-toe & Visual imaginary task (tell the road direc- & $20 / 80$ (emphasis on cognitive task) \\
& tion from home to the lab) & \\
\hline
\end{tabular}


employed in this research to screen children and to detect suspected DCD cases.

\section{Raven's Progressive Matrices test}

Raven's Progressive Matrices test is used to assess the general intelligence of children. This test is a non-verbal test for evaluating the problem-solving ability of children between the ages of 4 to 9 years old based on their prior information. In this test, the child is supposed to select the missing parts of pictures. This test was employed to select children with normal intelligence (Raven, Raven, Court, \& Cubero, 2001).

\section{Balance}

Y Balance Test which is a modified version of the Star Excursion Balance Test was employed to measure dynamic balance. For performing this test, the child has to stand on the non-dominant foot and try to touch three different points in different directions with their dominant foot. The participants performed six training trials to minimize the learning and warming up effect in each direction. They performed three trials in the anterior direction followed by another three trials in posterolateral directions and finally three trials in posteromedial directions. The highest score for every direction was recorded and after conversion to a normalized composite score, it was used in the analysis (Yam \& Fong, 2019). Previous studies have reported good reliability of this test (ICC $=.85-.91$; Barati, Bagheri, Azimi, Darchini, \& Nik, 2013). Normalized composite score on this test was calculated with this formula:

Normalized composite score $=[($ anterior + posterolateral + posteromedial)/(leg length*3)]*100.

In addition, Stork Balance Stand Test was used to test the static balance skill. For performing this test, the performer is supposed to stand on a flat surface one the non-dominant foot and raise the other foot as high as the knee level while both hands are placed to the sides of the body. The maximum time a performer stands in this position is recorded. This test is performed twice and the highest score is used in the analysis (Sharma \& Metgud, 2017). Scores were based on time. High testretest reliability has been reported or this test in the pediatric population (Chaouachi, Othman, Hammami, Drinkwater, \& Behm, 2014).

\section{Stages}

After the meeting and coordination arrangement with the school authorities, the teachers were asked to identify and introduce 7-9-year-old children who were suspected as DCD cases. Then, the parents of these children were invited and asked to complete the DCDQ'07 questionnaire. Based on the score derived from the DCDQ'07 questionnaire, children were selected as suspect to DCD (B criterion). Then, two experts examined the children individually by using the Bruininks-Oseretsky test in a quiet room and children whose score was one standard deviation below the mean (16th percentile, criterion A), were referred to a psychiatrist for possible exclusion. In addition, the IQ scores of children were assessed and those children within the normal level (IQ above 70) remained in the study. After the screening tests, 39 participants were selected. These children were randomly assigned into three groups: single-task, dual-task and control group. These children participated in static and dynamic balance tests in pre-test, prompt post-test (one day after 24 sessions) and a follow-up two months after they took part in the post-test stage.

\section{Statistical analysis}

Descriptive statistics including mean and standard deviations were calculated for all groups. Shapiro-Wilk test result showed that the dependent variables had a normal distribution; thus, inferential parametric statistical test including dependent $t$-test was used to test the within-group differences from the pre-test stage to the post-test and follow-up assessment. In order to investigate the possible initial differences between the groups and to control the pre-test, the one-way analysis of covariance (ANCOVA) was used. In addition, Bonferroni follow-up tests were employed to analyze the data. The entire hypothesis was tested at an alpha level set to .05 with two-tailed tests. The effect size was calculated by using partial eta-squared $\left(\eta_{\mathrm{p}}^{2}\right)$ and were based on the criteria of $\eta_{p}^{2}<.01$, small; $\eta_{p}^{2}=.06$, moderate; and $\eta_{\mathrm{p}}^{2}>.14$, large (Cohen, 1988). All analyses were performed by using SPSS software (Version 24.0; IBM, Armonk, NY, USA).

\section{Results}

Table 4 presents the means and standard deviation of some anthropometrical variables and results of the motor test. There was no significant difference between groups on these demographic characteristics at the start of the training stage.

\section{Interventions effects}

Table 5 shows the mean and standard deviations of the dependent variables based on the groups and stages. This table shows that the static and dynamic balance of the dual-task and single-task improved significantly from pre-test to post-test stages while no significant difference was present for the control group. In addition, the within-group differences between the pre-test and the post-test and follow-up stage is only significant 
Table 4

Characteristics of the participants (mean $\pm S D$ ) in the groups

\begin{tabular}{|c|c|c|c|c|}
\hline \multirow[b]{2}{*}{ Characteristics } & \multicolumn{3}{|c|}{ Group } & \multirow[b]{2}{*}{$p$} \\
\hline & Dual-task & Single-task & Control & \\
\hline Age (year) & $8.59 \pm 0.82$ & $8.61 \pm 0.83$ & $8.41 \pm 0.86$ & .801 \\
\hline Body height $(\mathrm{cm})$ & $129.53 \pm 4.84$ & $129.46 \pm 3.50$ & $128.76 \pm 3.19$ & .851 \\
\hline Body weight (kg) & $27.69 \pm 6.14$ & $27.38 \pm 4.29$ & $26.92 \pm 3.81$ & .921 \\
\hline Leg length $(\mathrm{cm})$ & $66.76 \pm 2.77$ & $66.69 \pm 2.32$ & $66.07 \pm 1.75$ & .701 \\
\hline Body mass index $\left(\mathrm{kg} / \mathrm{m}^{2}\right)$ & $16.40 \pm 3.01$ & $16.29 \pm 2.16$ & $16.17 \pm 1.69$ & .960 \\
\hline IQ (score) & $90.92 \pm 11.45$ & $89.30 \pm 6.01$ & $84.46 \pm 8.55$ & .171 \\
\hline DCDQ (score) & $44.92 \pm 5.02$ & $42.61 \pm 4.29$ & $43.53 \pm 4.33$ & .430 \\
\hline BOTMP (percentile) & $6.00 \pm 3.62$ & $7.00 \pm 3.60$ & $5.92 \pm 3.86$ & .711 \\
\hline \multicolumn{5}{|l|}{ Dominant foot } \\
\hline Right $(n, \%)$ & $4(30.8)$ & $3(23.1)$ & $4(30.8)$ & \\
\hline Left $(n, \%)$ & $9(69.2)$ & $10(76.9)$ & $9(69.2)$ & \\
\hline Sex & All male & All male & All male & \\
\hline
\end{tabular}

Note. $\mathrm{DCDQ}=$ Developmental Coordination Disorder Questionnaire; BOTMP $=$ Bruininks-Oseretsky Test of Motor Proficiency.

Table 5

Comparison of balance performance before (pre-test) and after (post-test and follow-up test) the intervention in the groups

\begin{tabular}{|c|c|c|c|c|c|}
\hline & \multicolumn{3}{|c|}{ Stage } & \multicolumn{2}{|c|}{$p$} \\
\hline & Pre-test & Post-test & Follow-up & Pre-test $\times$ post-test & Pre-test $\times$ follow-up \\
\hline \multicolumn{6}{|l|}{ Static balance } \\
\hline Dual-task & $5.43 \pm 0.34$ & $8.76 \pm 0.55$ & $6.42 \pm 0.42$ & $<.001$ & .001 \\
\hline Single-task & $5.53 \pm 0.48$ & $8.17 \pm 0.79$ & $5.98 \pm 0.55$ & $<.001$ & .053 \\
\hline Control & $5.41 \pm 0.41$ & $5.45 \pm 0.61$ & $5.36 \pm 0.44$ & .72 & .221 \\
\hline \multicolumn{6}{|c|}{ Dynamic balance } \\
\hline Dual-task & $80.07 \pm 6.54$ & $86.82 \pm 3.96$ & $84.12 \pm 3.61$ & $<.001$ & .002 \\
\hline Single-task & $79.46 \pm 5.34$ & $84.35 \pm 4.16$ & $81.48 \pm 3.89$ & .001 & .071 \\
\hline Control & $80.51 \pm 5.22$ & $80.56 \pm 5.09$ & $80.69 \pm 4.41$ & .65 & .591 \\
\hline
\end{tabular}

for the dual-task group for static and dynamic balance as well.

\section{Comparison of training programs}

The result of one-way ANCOVA test on the static balance scores in post-test stage using pre-test as a covariate showed a significant main effect for the training $\operatorname{program}\left(F(2,35)=107.48, p=.001, \eta_{\mathrm{p}}^{2}=.86\right)$. The result of Bonferroni post hoc test showed that there was a significant difference between the dual-task scores $(8.76 \pm 0.55)$ compared to single-task scores $(8.17 \pm 0.79, p=.029)$ and the scores in the control group $(5.45 \pm 0.61, p=.001)$. In addition, there was a significant difference between the single-task performance and the control group $(p=.001)$. The training program significantly improved the static balancing scores.

In the follow-up stage, the result of ANCOVA on static balance score by using the pre-test scores as covariate also shows a significant main effect for the training program $\left(F(2,35)=7.22, p=.001, \eta_{\mathrm{p}}^{2}=.47\right)$. The result of Bonferroni post hoc test showed that there was no significant difference between the dual-task group $(6.42 \pm 0.42)$ and single-task group $(5.98 \pm 0.55, p=.053)$, however, there were significant differences between dual-task group compared to the control group $(5.36 \pm 0.44, p=.001)$ but such differences were not found between the single-task and the control group $(p=.11)$.

The result of one-way ANCOVA test on the mean of dynamic balance scores in post-test stage by including 
pre-test scores as a covariate showed a significant main effect for the training program $(F(2,35)=45.90$, $\left.p=.001, \eta_{\mathrm{p}}^{2}=.72\right)$. The result of Bonferroni post hoc test indicated that the significant difference $(p=.029)$ was between the group with the dual-task programme $(86.82 \pm 3.96)$ compared to the group with single-task programme $(84.35 \pm 4.16)$ and the control group $(80.56 \pm 5.09, p=.001)$. In addition, there was a significant difference between the group with the singletask programme and the control group $(p=.001)$. The training program significantly improved the dynamic balancing scores (Table 4).

A similar analysis was performed for the follow-up scores. The result of ANCOVA in follow-up stage on dynamic balance score by using the pre-test scores as covariate also showed a significant main effect for the training program $(F(2,35)=9.93, p=.001$, $\left.\eta_{\mathrm{p}}^{2}=.360\right)$. The result of the Bonferroni post hoc test showed that there was a significant difference between groups with dual-task programme $(84.12 \pm 3.61)$ and single-task programme $(81.48 \pm 3.89, p=.03)$ and control group $(80.69 \pm 4.41, p=.001)$. The training program significantly improved the dynamic balance scores in the dual-task condition; however, there was no significant difference between the group with single-task programme compared to the control group.

\section{Discussion}

The present research was designed to compare the effect of single-task versus dual-task training on static and dynamic balance skills of children with DCD. The findings of this research indicated that the experimental groups in both training conditions significantly improved their balance skills in dynamic and static skills in the post-test stage. In other words, specific task training in single and dual-task training improved the static and dynamic balancing skills of DCD children. This finding is similar to the findings of (Fong, Guo, Liu, et al., 2016; Fong, Tsang, \& Ng, 2012; Giagazoglou et al., 2015) who demonstrated that balance intervention training results in improvement of balance skills in DCD children. The training protocol employed in this research included a collection of physical training and progressively increasing repetitive dynamic and static exercises with the aim of improving the balance skills of DCD children. The training program was designed to meet the suggestions made in the task-oriented condition with an emphasis on improving balance skills primarily practised in a close environment shifted to more difficult tasks performed in open environment adopted by Gentile view of classifying motor skill tasks (Magill, 2007).
Thus, as the skill level of children improved, the sessions and training level shifted from the simple form suggested in Gentile's two-dimensional classification system to a more difficult condition. This criterion was assumed to have a positive effect on the training protocol to improve the dynamic and static balance skills of DCD children.

Another finding was the significant improvement difference between the single and dual-task on static and dynamic balance scores. Indeed, the dual-task group showed greater improvement in the static and dynamic balance performance. It can be said probably focusing on the cognitive and balancing task resulted in improvement of balancing skills in dynamic and static balance performances. This result also confirms the findings of some previous studies demonstrating that practising dual-task skills is more effective than the single-tasks training for improving balancing skills (Chen et al., 2012; Choi et al., 2015; Elhinidi et al., 2016; Fernandes, Rocha, Santos, \& Tavares, 2015; Silsupadol et al., 2006; Zijlstra, Ufkes, Skelton, Lundin-Olsson, \& Zijlstra, 2008). There are several explanations for observing such findings; some researchers contribute this discrepancy of effectiveness to the insufficiency of attention in single-tasks; during the dual-task training, the interaction of motor task and cognitive demand on the performer increases the attention demands which in turn results in an improvement in balance execution (Choi et al., 2015). In fact, it seems that DCD children show poor performance on such tasks due to a decline in their attention resources when information processing on a motor task (Cherng et al., 2009) and during the performance of a dual-task, the secondary task results in improvement of cognitive resources that finally causes better skill execution on motor tasks.

There seems to be a beneficial effect of the simultaneous use of a cognitive task when performing other tasks for children who show poor motor performance. Particularly, the DCD children focus on the movement at hand when performing a single-task which in turn causes disturbance in the dynamics of the self-organizing movement system (Schott, El-Rajab, \& Klotzbier, 2016).

From the physiological point of view, the improvement demonstrated by the DCD children in a dualtask group compared to the single-task group may be attributed to the strong effect of the dual-task motor program on the activation of a substitution path that includes the cerebellum, pre-motor cortex and lateral pre-motor cortex. In this path, the cerebellum is in charge of movement timing. Also, when a movement is facilitated by the deep sensory signal related to that movement, the pre-motor cortex is in charge of scaling the physical activity. This means that triggering 
and activating these structures may compensate for ineffective basal ganglions function (Elhinidi et al., 2016). In other words, probably using dual-task in the prolonged interventional program may lead to neural plasticity in the brain. Following this activation, neural processing (sensory-motor, cortical, cerebellum) and circuits of the cerebellar cortex are improved; as a result, balance skill is improved (Holschneider, Yang, Guo, \& Maarek, 2007).

Furthermore, research results have indicated that managing dual-task skills such as walking and maintaining balance requires executive functional processes. The results of the research show that there is an association between the executive functions and premotor cortex. In addition, it is evident that the premotor cortex function improves as a result of cognitive and motor interventions. This type of plasticity by the premotor cortex and its association with the executive functions may explain why dual-task compared to the singletask training resulted in more improvement in static and dynamic balance skills. In fact, maintaining balance is a complex task that involves several processes of which cognitive process is one of them (Fraser et al., 2017). This was the case in the present research; cognitive training in the dual-task group resulted in the improvement of cognitive processes that eventually caused superior balance skill execution in this group compared to the single-task group.

Another result of the present research was that there were no significant differences between the single and dual-task in static balance scores in the follow-up stage while there the dual-task group showed the superiority of performance in dynamic balance scores compared to the single-task and control group. It seems that during the execution of a dynamic balance task, due to the more cognitively involved complex mechanisms, training the dual-task may have more durable effects than the single-task training.

The method adopted by this research is an innovative approach since the training method was the first of its kind to examine the effect of a dual-task balance training program on the improvement of static and dynamic balance of DCD children. However, the researcher acknowledges the limitations of the protocol due to the sample size used in every training group for generalizing the findings to the population of DCD children. More research with a larger sample size is needed to make a firm recommendation for the use of these training methods. Considering the lack of finding significant differences between the two experimental groups in static balance in follow-up, it is likely that a prolonged intervention may have shown different findings in regard to the effectiveness of dual-task. In addition, considering the fact that the study design was based on the most related views in this regard (Plummer-D’Amato et al., 2012; Silsupadol et al., 2006), more researches were suggested by introducing the cognitive task before or after the balance task. Since previous researches have indicated that dual-task training effect is dependent on age and adult participants demonstrate more benefit from performing this type of training (Swan, Otani, Loubert, Sheffert, \& Dunbar, 2004), more research is needed to compare the effect of this task-oriented training in DCD adults versus DCD children.

In the present study, the effects were assessed at the level of the motor performance only, without the use of methods at the level of biomechanical, muscular or neurobehavioral analyses that would contribute to a better understanding of mechanisms underlying effects of dual-task balance. To confirm findings and valid results it is needed to investigate the underlying mechanisms.

\section{Conclusions}

The result of this research showed that balance training program with single or dual-task conditions resulted in the improvement of static and dynamic balancing skills in DCD children. These effects remained even after two months of terminating the program in the dual-task training condition. In addition, based on the findings of the present research, the dual-task training condition focusing on balance and cognitive tasks may improve the skills of children on performing static and dynamic balance more significantly than the single-task.

Results related to the effect of dual-task training should be expressed with caution. Because the singletask balance program also improved significantly both static and dynamic balance in the post-test and followup. Improvement in balance abilities can be a result of intensive practising of balance exercises without applying the dual-task strategy.

\section{Acknowledgments}

We would like to thank Moira van Leeuwen for careful reading of the manuscript and her suggestions. We would also like to thank all of the participants, their families and the schools who took part in this study.

\section{Conflict of interest}

There were no conflicts of interest. 


\section{References}

Andersson, G., Hagman, J., Talianzadeh, R., Svedberg, A., \& Larsen, H. C. (2002). Effect of cognitive load on postural control. Brain Research Bulletin, 58, 135-139.

Barati, A. H., Bagheri, A., Azimi, R., Darchini, M. A., \& Nik, H. N. (2013). Comparison balance and footprint parameters in normal and overweight children. International Journal of Preventive Medicine, 4, S92-S97.

Bruininks, R. H. (1978). Bruininks-Oseretsky Test of Motor Proficiency. Circle Pines, MN: American Guidance Service.

Bruininks, R., \& Bruininks, B. (2005). Bruininks-Oseretsky Test of Motor Proficiency (2nd ed.). Minneapolis, MN: NCS Pearson.

Caçola, P., \& Lage, G. (2019). Developmental Coordination Disorder (DCD): An overview of the condition and research evidence. Motriz: Revista de Educação Física, 25, e101923.

Chaouachi, A., Othman, A. B., Hammami, R., Drinkwater, E. J., \& Behm, D. G. (2014). The combination of plyometric and balance training improves sprint and shuttle run performances more often than plyometric-only training with children. Journal of Strength and Conditioning Research, 28, 401-412.

Cheldavi, H., Shakerian, S., Boshehri, S. N. S., \& Zarghami, M. (2014). The effects of balance training intervention on postural control of children with autism spectrum disorder: Role of sensory information. Research in Autism Spectrum Disorders, 8, 8-14.

Chen, Y.-Y., Chen, C.-H., Wang, C.-C., Liaw, L.-J., Guo, L.-Y., \& Wu, W.-L. (2012). Comparison of dynamic balance under dual and simple task conditions in children with attention deficit hyperactivity disorder. Journal of Physical Therapy Science, 24, 633-637.

Cherng, R.-J., Liang, L.-Y., Chen, Y.-J., \& Chen, J.-Y. (2009). The effects of a motor and a cognitive concurrent task on walking in children with developmental coordination disorder. Gait and Posture, 29, 204-207.

Choi, J. H., Kim, B. R., Han, E. Y., \& Kim, S. M. (2015). The effect of dual-task training on balance and cognition in patients with subacute post-stroke. Annals of Rehabilitation Medicine, 39, 81-90.

Cohen, J. (1988). Statistical power analysis for the behavioral sciences (2nd ed.). Hillsdale, NJ: Lawrence Erlbaum Associates.

Elhinidi, E. I. M., Ismaeel, M. M. I., \& El-Saeed, T. M. (2016). Effect of dual-task training on postural stability in children with infantile hemiparesis. Journal of Physical Therapy Science, 28, 875-880.

Fernandes, Â., Rocha, N., Santos, R., \& Tavares, J. M. R. (2015). Effects of dual-task training on balance and executive functions in Parkinson's disease: A pilot study. Somatosensory \& Motor Research, 32, 122-127.

Fong, S. S., Guo, X., Cheng, Y. T., Liu, K. P., Tsang, W. W., Yam, T. T., ... Macfarlane, D. J. (2016). A novel balance training program for children with developmental coordination disorder: A randomized controlled trial. Medicine, 95, e3492.

Fong, S. S., Guo, X., Liu, K. P., Ki, W., Louie, L. H., Chung, R. C., \& Macfarlane, D. J. (2016). Task-specific balance training improves the sensory organisation of balance control in children with developmental coordination disorder: A randomised controlled trial. Scientific Reports, 6, 20945.

Fong, S. S., Tsang, W. W., \& Ng, G. Y. (2012). Taekwondo training improves sensory organization and balance control in children with developmental coordination disorder: A randomized controlled trial. Research in Developmental Disabilities, 33, 85-95.

Fraser, S. A., Li, K. Z.-H., Berryman, N., Desjardins-Crépeau, L., Lussier, M., Vadaga, K., ... Bosquet, L. (2017). Does combined physical and cognitive training improve dual-task balance and gait outcomes in sedentary older adults? Frontiers in Human Neuroscience, 10, 688.

Giagazoglou, P., Sidiropoulou, M., Mitsiou, M., Arabatzi, F., $\&$ Kellis, E. (2015). Can balance trampoline training promote motor coordination and balance performance in children with developmental coordination disorder? Research in Developmental Disabilities, 36, 13-19.

Hamacher, D., Hamacher, D., \& Schega, L. (2014). A cognitive dual task affects gait variability in patients suffering from chronic low back pain. Experimental Brain Research, 232, 3509-3513.

Holschneider, D., Yang, J., Guo, Y., \& Maarek, J.-M. (2007). Reorganization of functional brain maps after exercise training: Importance of cerebellar-thalamic-cortical pathway. Brain Research, 1184, 96-107.

Magill, R. A. (2007). Motor learning and control: Concepts and applications (8th ed.). New York, NY: McGraw-Hill.

Makizako, H., Doi, T., Shimada, H., Yoshida, D., Tsutsumimoto, K., Uemura, K., \& Suzuki, T. (2012). Does a multicomponent exercise program improve dual-task performance in amnestic mild cognitive impairment? A randomized controlled trial. Aging Clinical and Experimental Research, 24, 640-646.

Mandich, A. D., Polatajko, H. J., Macnab, J. J., \& Miller, L. T. (2001). Treatment of children with Developmental Coordination Disorder. Physical \& Occupational Therapy in Pediatrics, 20(2-3), 51-68.

Plummer-D’Amato, P., Cohen, Z., Daee, N. A., Lawson, S. E., Lizotte, M. R., \& Padilla, A. (2012). Effects of once weekly dual-task training in older adults: A pilot randomized controlled trial. Geriatrics \& Gerontology International, 12, 622-629.

Raven, J., Raven, Court, J. H., \& Cubero, N. S. (2001). Raven: matrices progresivas: escalas Color (CPM), General (SPM), Superior (APM): manual [Raven: Progressive matrices: Scales Coloured (CPM), Standard (SPM), Advanced (APM): Manual] (3rd ed.). Madrid, Spain: TEA Ediciones.

Revie, G., \& Larkin, D. (1993). Task-specific intervention with children reduces movement problems. Adapted Physical Activity Quarterly, 10, 29-41.

Schott, N., El-Rajab, I., \& Klotzbier, T. (2016). Cognitivemotor interference during fine and gross motor tasks in children with Developmental Coordination Disorder (DCD). Research in Developmental Disabilities, 57, 136-148.

Sharma, P., \& Metgud, D. (2017). Assessment of static and dynamic balance in overweight and obese children with and without flatfoot: A cross-sectional study. Indian Journal of Health Sciences and Biomedical Research, 10, 173-177. 
Silsupadol, P., Siu, K.-C., Shumway-Cook, A., \& Woollacott, M. H. (2006). Training of balance under single-and dualtask conditions in older adults with balance impairment. Physical Therapy, 86, 269-281.

Smits-Engelsman, B., Schoemaker, M., Delabastita, T., Hoskens, J., \& Geuze, R. (2015). Diagnostic criteria for DCD: Past and future. Human Movement Science, 42, 293-306.

Smits-Engelsman, B., Vincon, S., Blank, R., Quadrado, V. H., Polatajko, H., \& Wilson, P. H. (2018). Evaluating the evidence for motor-based interventions in developmental coordination disorder: A systematic review and metaanalysis. Research in Developmental Disabilities, 74, 72-102.

Speedtsberg, M. B., Christensen, S. B., Andersen, K. K., Bencke, J., Jensen, B. R., \& Curtis, D. J. (2017). Impaired postural control in children with developmental coordination disorder is related to less efficient central as well as peripheral control. Gait and Posture, 51, 1-6.

Strang, A. J., Haworth, J., Hieronymus, M., Walsh, M., \& Smart, L. J. (2011). Structural changes in postural sway lend insight into effects of balance training, vision, and support surface on postural control in a healthy population. European Journal of Applied Physiology, 111, 1485-1495.

Sugden, D. (2007). Current approaches to intervention in children with developmental coordination disorder. Developmental Medicine \& Child Neurology, 49, 467-471.

Swan, L., Otani, H., Loubert, P. V., Sheffert, S. M., \& Dunbar, G. L. (2004). Improving balance by performing a secondary cognitive task. British Journal of Psychology, 95, 31-40.

Wild, L. B., de Lima, D. B., Balardin, J. B., Rizzi, L., Giacobbo, B. L., Oliveira, H. B., ... Bromberg, E. (2013). Characterization of cognitive and motor performance during dual-tasking in healthy older adults and patients with Parkinson's disease. Journal of Neurology, 260, 580-589.

Wilson, B. N., Crawford, S. G., Green, D., Roberts, G., Aylott, A., \& Kaplan, B. J. (2009). Psychometric properties of the revised Developmental Coordination Disorder Questionnaire. Physical \& Occupational Therapy in Pediatrics, 29, 182-202.

Wilson, P. H. (2005). Practitioner review: Approaches to assessment and treatment of children with DCD: An evaluative review. Journal of Child Psychology and Psychiatry, 46, 806-823.

Yam, T. T. T., \& Fong, S. S. M. (2019). Y-Balance Test performance and leg muscle activations of children with developmental coordination disorder. Journal of Motor Behavior, 51, 385-393.

Zijlstra, A., Ufkes, T., Skelton, D., Lundin-Olsson, L., \& Zijlstra, W. (2008). Do dual tasks have an added value over single tasks for balance assessment in fall prevention programs? A mini-review. Gerontology, 54, 40-49.

Zwicker, J. G., Missiuna, C., Harris, S. R., \& Boyd, L. A. (2012). Developmental coordination disorder: A pilot diffusion tensor imaging study. Pediatric Neurology, 46, 162-167. 